\title{
The Association Between Serum Biomarker Levels and Clinical Outcomes among Acute Ischemic Stroke Patients
}

\author{
Retnaningsih ${ }^{1}$, Ben Asiel Padang ${ }^{1}$, Windri Kartikasari ${ }^{1}$, Christina \\ Roseville Lasma Aritonang ${ }^{1}$, Amin Husni ${ }^{1,2}$ and Santoso Jaeri ${ }^{3 *}$ \\ ${ }^{1}$ Department of Neurology, Dr. Kariadi Hospital/Faculty of Medicine, Diponegoro \\ University, Semarang, Indonesia \\ ${ }^{2}$ Department of Anatomy and Histology, Faculty of Medicine, Diponegoro \\ University, Semarang, Indonesia \\ ${ }^{3}$ Department of Medical Biology and Biochemistry, Faculty of Medicine, Diponegoro \\ University, Semarang, Indonesia \\ *Corresponding Author: Santoso Jaeri, Department of Medical Biology and \\ Biochemistry, Faculty of Medicine, Diponegoro University, Semarang, Indonesia.
}

Received: April 22, 2021

Published: June 11, 2021

(C) All rights are reserved by Retnaningsih., et al.

\section{Abstract}

Background: Ischemic brain damage results in the release of some of the neurons or glia-specific biomarkers including S100 calcium-binding protein B (S100B), glial fibrillary acidic protein (GFAP), and neuron-specific enolase protein (NSE). These biomarkers are currently being used in neuroimaging studies in the management of stroke patients. Unfortunately, the association of these biomarkers and clinical outcome among acute ischemic stroke patients are still in debate. This study determines the association of serum S100B, NSE, and GFAP levels at 72 hours onset with the changes of the NIHSS score between the onset of day 3 and day 7 in acute ischemic stroke patients.

Methodology: A prospective observational cohort study among 42 subjects with acute ischemic stroke who met the inclusion and exclusion criteria. The serum S100B, GFAP, and NSE levels were measured using enzyme-linked immunosorbent assay (ELISA) from venous blood at 72 hours onset of ischemic stroke. NIHSS scores were measured on days 3 and 7 . At the end of the study, the subjects were classified based on the improvement of clinical improvement into two groups; NIHSS score improved (n = 18), and not improved $(n=24)$. The differences of the S100B, GFAP, and NSE levels among both groups were analyzed using the Mann-Whitney $\mathrm{U}$ test, continued with ROC curve analysis to find out the cut-off of S100B. The association between S100B and clinical outcome was analyzed using the Chi-square test.

Results: There was a significant difference on S100B levels ( $p=0.014$ ), but no difference on serum NSE and GFAP levels among both groups ( $p=0.446$ and $p=0.205$ ), there was significant association between serum S100B levels and the improvement of clinical outcome in ischemic stroke patients $(\mathrm{p}=0.007, \mathrm{HR}=6.000,95 \% \mathrm{CI}=1.560-23.072)$.

Conclusion: There is a significant association between the 72 hours onset serum S100B level and the improvement of clinical outcome among acute ischemic stroke patients. The patients who have serum S100B level $608 \mathrm{ng} / \mathrm{mL}$ or less have a chance to get improved clinical outcome are six times compared to the patients who have serum S100B level more than $608 \mathrm{ng} / \mathrm{mL}$.

Keywords: Biomarkers; S100B; GFAP; NSE; NIHSS; Acute Ischemic Stroke 


\section{Introduction}

Stroke is a very serious neurological disease. Stroke causes disability and mortality resulting in a decrease in patient's quality of life. Besides, stroke is the second cause of death in the world according to WHO [1]. In Indonesia, the mortality rate is $15.4 \%$, or approximately 99 out of 100,000 people, and causing disability in 685 of 100,000 people $[2,3]$.

The severity of stroke is measured using the National Institutes of Health Stroke Scale (NIHSS). NIHSS is one of the assessment tools that quantitatively measures the neurological deficits of stroke patients who are reliable and valid in predicting long-term clinical outcomes.

Brain injury resulted from an ischemic stroke causes neuronal damage followed by the release of specific neuronal and glial cell biomarkers such as S100B protein, glial fibrillary acidic protein (GFAP), myelin basic protein (MBP), and neuron-specific enolase (NSE). The release of these biomarkers occurred after the damage of neurons, glia cells, and blood-brain barrier due to ischemic cascades. The previous study demonstrated that there is a correlation between the serum levels of some neuronal and glial cell biomarkers with the degree of brain tissue damage. Thus, there is potency in the ability of these biomarkers to predict the clinical outcome of acute ischemic stroke patients [1,5-7]. Some biomarkers as a consequence of ischemic brain damage are currently being used in the neuroimaging studies such as computed tomography (CT) and magnetic resonance imaging (MRI) to measure the degree of ischemic brain damage in the management of stroke patients, despite the association of these biomarkers on the clinical outcome still in debate. ${ }^{1}$ Some studies demonstrated that the quantification of serum concentrations of both NSE and S100B after acute stroke may be clinically relevant to predict the functional neurological outcome, another study showed that only S 100B protein level is associated with stroke volume, neurological status at admission and functional outcome.

The controversy regarding the best biomarkers to predict the clinical outcome of ischemic stroke lead us to confirm the association of S100B, GFAP, and NSE with the short-term clinical outcome of ischemic stroke.

\section{Materials and Methods}

The study was conducted among inward patients of the Department of Neurology Kariadi Hospital, Semarang Indonesia. Subjects were patients diagnosed with acute ischemic stroke, admitted to those hospitals that fulfill the inclusion criteria. On day seven of onset, the NIHSS score was re-measured.

The data was then collected, calculated, and analyzed statistically using SPSS for Windows version 16 . The analysis was carried out in two stages, first was descriptive analysis to determine the basic characteristics of the subjects and the second was analytic analysis to determine the association between variables. The normality test of numerical scale data was performed by using the SaphiroWilk test. The bivariate analysis to determine the differences of the S100B, GFAP, and NSE levels among both groups were analyzed using the Mann-Whitney $U$ test, continued with ROC curve analysis to find out cut off of S100B. The association between S100B and clinical outcome was analyzed using the Chi-square test.

\section{Results and Discussion}

The association of biomarkers resulted from the ischemic brain damages on the clinical outcome still in debate [1]. Some studies demonstrated that the quantification of serum concentrations of both NSE and S100B after acute stroke may be clinically relevant to predict the functional neurological outcome, another study showed that only S 100B protein level is associated with stroke volume, neurological status at admission and functional outcome. Herewith we provide the evidence of the association of S100B, GFAP, and NSE with short-term clinical outcome of ischemic stroke.

In the brain, S100B can be found in glial cells such as astrocytes, oligodendrocytes, Schwann cells, ependymal cells, and Muller retinal cells. The mean level of S100B is $0.05 \mu \mathrm{g} / \mathrm{L}$. There is no difference level of S100B between men and women. S100B levels in normal people range from 0.02 to $0.15 \mu \mathrm{g} / \mathrm{L} .14$ [8], When structural damage such as infarction in the cytosolic glia cells and Schwann cells occurs, S100B is released into the cerebrospinal fluid (CSF) and blood. Several studies reported that S100B was a marker of blood-brain barrier damage, and its concentration in CSF is 40 times higher than in serum. In the first few hours after the onset of stroke, a high serum level of S100B showed blood-brain barrier damage rather than a marker for infarct area. Previous studies have shown that S100B increased significantly after stroke onset compared to the control group and correlated with infarct volume, stroke severity, and functional outcome [4].

GFAP is an intermediate filament III protein that is uniquely found in astrocytes in the central nervous system (CNS), non-my- 
elinated Schwann cells in the peripheral nervous system, and enteric glial cells. GFAP gene activation and protein induction appear to play an important role in astroglial cell activation after CNS injury and neurodegeneration. GFAP is released rapidly in wide brain parenchyma bleeding that causes direct cell damage, whereas it is late detected in ischemic stroke, where necrosis and cellular disintegration occur 6-12 hours after symptom onset [9]. GFAP levels in healthy individuals are consequently very low, usually not exceeding the lower detection limit of the tests used. Furthermore, GFAP levels in the blood proved to correlate with the severity and outcomes after traumatic brain injury $[10,11]$. The normal GFAP in healthy individuals is $<0.15 \mu \mathrm{g} / \mathrm{L}[10]$.

Enolases are intracellular enzymes that catalyze dehydration of 2-phosphate-glycolysis to phosphoenolpyruvate in the glycolytic pathway, a process that converts glucose into pyruvate, which allows the formation of high energy ATP and NADH $[12,13]$. Increased NSE activity in serum and CSF has been reported to correlate with brain injury [13]. This biomarker will be released intracellularly and extracellularly to CSF and blood if CNS injuries, accompanied by neuronal tissue damage occur [13]. It is released into the systemic circulation after neurological damage, including ischemic stroke, cerebral hemorrhage, and traumatic brain injury or hypoxia. Therefore, a sudden increase in NSE levels in the blood provides a diagnostic and prognostic marker of brain damage and brain blood barrier damage [14].

This study comprised 42 subjects, 23 male and 19 female. The mean age was $61.67 \pm 12.3$ years old, with the youngest was 17 years and the oldest was 84 years. In the clinical characteristics, the majority of subjects did not suffer from diabetes mellitus (DM) $(83.3 \%)$. Most of the subjects suffered from hypertension (76.2\%), and dyslipidemia (85.7\%). There were 9.5\% subjects with a single lacunar, $76.2 \%$ with multiple lacunars, $9.5 \%$ with a single territorial, and $4.8 \%$ with mixed infarction patterns. Most of them were non-obese (78.6\%), while $21.4 \%$ of subjects are obese. The characteristics of subjects based on the improvement of clinical outcome are depicted in table 1 . Table 1 demonstrated that there is no significant association in the most of characteristics except for the BMI status.

Regarding the characteristics of the subjects, in our study, male subjects (54.8\%) were more than women (45.2\%). This is consistent with the literature which stated that the incidence of stroke in
Table 1: The demographic and clinical characteristics of subjects based on clinical outcome improvement.

\begin{tabular}{|c|c|c|c|}
\hline \multirow[b]{2}{*}{ Variable } & \multicolumn{2}{|c|}{ Clinical Outcome } & \multirow[b]{2}{*}{$\mathbf{p}$} \\
\hline & $\begin{array}{l}\text { Improved } \\
\qquad(n=18)\end{array}$ & $\begin{array}{l}\text { Not improved } \\
\qquad(n=24)\end{array}$ & \\
\hline \multicolumn{4}{|l|}{ Gender } \\
\hline Male & $8(44,4)$ & $15(62,5)$ & 0,245 \\
\hline Female & $10(55,6)$ & $9(37,5)$ & \\
\hline \multicolumn{4}{|l|}{ Age } \\
\hline$<65$ & $13(72,2)$ & $12(50)$ & 0,147 \\
\hline$\geq 65$ & $5(27,8)$ & $12(50)$ & \\
\hline \multicolumn{4}{|l|}{ Diabetes Mellitus } \\
\hline Yes & $2(11,1)$ & $5(20,8)$ & 0,679 \\
\hline No & $16(88,9)$ & $19(79,2)$ & \\
\hline \multicolumn{4}{|l|}{ Hypertension } \\
\hline Yes & $16(88,9)$ & $16(66,7)$ & 0,147 \\
\hline No & $2(11,1)$ & $8(33,3)$ & \\
\hline \multicolumn{4}{|l|}{ Dyslipidemia } \\
\hline Yes & $16(88,9)$ & $20(83,3)$ & 0,685 \\
\hline No & $2(11,1)$ & $4(16,7)$ & \\
\hline \multicolumn{4}{|l|}{ Infarct size } \\
\hline Lacunar, single & $3(16,7)$ & $1(4,2)$ & \\
\hline Lacunar, multiple & $14(77,8)$ & $18(75)$ & 0,970 \\
\hline Single territory & $1(5,6)$ & $3(12,5)$ & \\
\hline Mixed & $0(0)$ & $2(8,3)$ & \\
\hline \multicolumn{4}{|l|}{ BMI status } \\
\hline Non obese & $11(61,1)$ & $22(91,7)$ & $0,025^{*}$ \\
\hline Obese & $7(38,9)$ & $2(8,3)$ & \\
\hline
\end{tabular}

$* \mathrm{p}<0.005$

women aged 45-54 years was lower than men even though with increasing age this difference decreased, even at the age of 85 years the incidence of stroke in black women was almost three times higher than men [14]. This indicated that there was no relationship between gender and neurological clinical outcomes. The study of Koichiro Maeda., et al. found that women had longer hospital stays and worse clinical outcomes than men. Women had older stroke onset $(\mathrm{p}=0.0001)$, more frequent incidence of cardioembolism $(p=0.0009)$, more frequent of hypertension $(p=0.0267)$, more 
frequent dyslipidemia ( $\mathrm{p}=0.0001)$, longer time needed to be admitted to the hospital $(\mathrm{p}=0.026)$, higher initial NIHSS score $(\mathrm{p}=$ $0.0010)$, and longer duration of hospital stay $(p=0.010)$ compared to men.

Our study found that there was no association between age and neurological clinical outcomes ( $p=0.147$ ) in acute ischemic stroke patients. The results of the logistic regression test showed that the age of younger than 65 years as a predictor of improvement in the NIHSS score (15.334 times) in acute ischemic stroke patients. The study of Shrestha also stated that age did not have a significant effect on neurological clinical outcomes of acute ischemic stroke patients. The mean age of the subjects was $67.04 \pm 13.39$ years. Age did not significantly influence bivariate analysis but multivariate logistic regression analysis showed it as a good predictor of ischemic stroke output [15].

Our study reported that there was no association between DM and neurological clinical outcomes of acute ischemic stroke patients ( $\mathrm{p}=0.480$ ). Jia., et al. also reported that subjects with DM had a significantly higher incidence of death, dependency, and stroke recurrence rates compared to subjects without DM. DM was an independent risk factor for mortality and dependence $(\mathrm{OR}=1.23$, confidence interval $=1.10-1.37$ ) in ischemic stroke patients after 6 months of onset. ${ }^{35}$ This study had different results from the literature which stated that DM was associated with neurological clinical outcomes of acute ischemic stroke patients. The condition of hyperglycemia will contribute to poor outcomes in patients with acute ischemic stroke. Hyperglycemia that occurs in acute ischemic stroke is a response mechanism to metabolic stress which results in severe neuronal tissue damage of the brain which will affect the clinical outcome of acute ischemic stroke patients. Nevertheless, there are many other factors besides hyperglycemia that are more dominant in influencing the clinical outcome of acute ischemic stroke patients.

Our study indicated that there was no association between hypertension and neurological clinical outcome of acute ischemic stroke patients $(\mathrm{p}=0.147)$. The result of the logistic regression test showed that hypertension was a predictor of improvement in the NIHSS score of (19.671 times) in acute ischemic stroke patients ( $p$ $=0,250$ ). Nayak., et al. in India reported a comparison of clinical outcomes of acute ischemic stroke patients with and without hypertension. The mortality and dependency rates of acute ischemic stroke patients at the time of discharge were similar between patients with and without hypertension. Whereas, it showed a higher dependency ratio in acute ischemic stroke patients with hypertension compared with patients without hypertension in the longterm output at 12 months and 18 months [16].

The results of this study did not find an association between the condition of dyslipidemia and the neurological clinical outcome of acute ischemic stroke patients $(\mathrm{p}=0.685)$. A large-scale study in China conducted by Xu Tian., et al. involving 1,568 subjects who obtained serum total cholesterol and HDL cholesterol were significantly associated with the outcome of acute ischemic stroke patients [13]. Kwon., et al. who examined the predictive factors of neurological deterioration in lacunar infarct stroke patients indicated that DM, systolic blood pressure, triglycerides, total cholesterol, LDL cholesterol, and homocysteine associated with neurological deterioration on the $7^{\text {th }}$ day of onset based on bivariate analysis. Based on the highest quartile multiple logistic regression analysis, triglyceride levels were an independent predictor of neurological deterioration in patients with lacunar infarct stroke $\mathrm{OR}=$ 11.46 , confidence interval $=1.07-122.87, \mathrm{p}=0.044$ ).

Our study indicated that there was a significant relationship between BMI status and neurological clinical outcome of acute ischemic stroke patients $(p=0.025)$. The results of the logistic regression test showed that the non-obese status was a risk factor for the improvement of the NIHSS score of 0.672 times in acute ischemic stroke patients $(\mathrm{p}=0.018)$. Bas., et al. reported that obese patients had a better initial NIHSS score, but had similar clinical symptoms and output, also the risk of hemorrhagic transformation or death between obese and non-obese patients [17]. There was no correlation between the infarct area and neurological clinical outcome in this study ( $p=0.970)$. This was inconsistent with previous studies which stated that infarct size was associated with neurological clinical outcomes of acute ischemic stroke patients. This is probably because the infarct location is more dominantly affecting stroke outcome compared to the infarct size factor alone.

The mean NIHSS score of the third onset day was 8.09 (SD = 4.9), with the lowest was 3 and the highest was 18. 14 subjects had mild NIHSS and 28 subjects had moderate NIHSS. 18 subjects experienced improvements in the NIHSS score (42.8\%), while 24 subjects did not experience an improvement in the NIHSS score $(57.2 \%)$. 
The analysis data then continued with the bivariate analysis to determine the differences of the S100B, GFAP, and NSE levels among both groups and the results of these analyses demonstrated in table 2. Table 2 provide evidence that there is a significant difference of serum S100B levels with the improvement of clinical outcome in ischemic stroke patients, but no for NSE and GFAP levels.

The median serum S100B level was $894 \mathrm{ng} / \mathrm{L}$ with the lowest of $194.2 \mathrm{ng} / \mathrm{L}$ and the highest of 4,097 ng/L. Our study found that there is a significant association between serum S100B levels and the improvement of clinical outcome. The patients who have serum S100B level $608 \mathrm{ng} / \mathrm{mL}$ or less have a chance to get improved clinical outcome are six times compared to the patients who have serum S100B level more than $608 \mathrm{ng} / \mathrm{mL}$. This is similar to Foerch., et al. which stated that S100B levels measured at 48 and 72 hours after onset provided the best predictive value for the functional outcome as measured by mRS 6 months after onset [13].

However, there was no significant relationship between serum NSE levels and NIHSS changes in acute ischemic stroke patients. Zaheer., et al. found a positive correlation between NSE concentration on day 1 and infarct volume determined by CT scan. A negative correlation was found between GCS and NSE concentration on day 1. There was a positive correlation between NSE levels on day 1 and functional neurological outcomes assessed by mRS on day 30 . Motiani., et al. also obtained similar results, where there was a significant positive correlation between increased serum NSE levels and poor neurological outcomes, which were clinically determined by MRS scores and NIHSS. The NIHSS had a strong positive correlation with serum NSE levels on day 1 and day 7. on the other hand, Haupt., et al. provided insignificant results between NSE and neurological clinical outcomes. Furthermore, the NSE value alone cannot distinguish significantly between the two outcome groups. In our study, the blood sampling of NSE might cause less specific results to assess nerve damage and compare with the clinical outcome than if NSE was taken through CSF. However, CSF sampling is difficult because a lumbar puncture is often contraindicated in acute neurological injury, such as ischemic stroke, unlike CSF plasma or serum sampling. Several studies that correlate NSE with clinical outcome using mRS had significant results. Whereas in our study, the clinical neurological outcome was assessed using NIHSS on days 3 and 7 , so that neurological changes could not be seen and less able to describe clinical outcomes. This study might produce more significant results if reassessed at day 30, 3 months, and 6 months [7]. Although the relationship between NSE and neurological clinical outcome changes (NIHSS) was statistically insignificant in this study, it was found that the higher the NSE level, the worse

Table 2. The comparison of serum biomarkers level on group with and without improvement of clinical outcome.

\begin{tabular}{lccc}
\hline & \multicolumn{2}{c}{ Clinical Outcome } & \\
\cline { 2 - 3 } & $\begin{array}{c}\text { Improved } \\
(\mathbf{n = 1 8})\end{array}$ & $\begin{array}{c}\text { Not Improved } \\
\text { (n= 24) }\end{array}$ & \\
\hline Serum S100B & $840.15(194.2$ & $-1332.50(243-$ & \\
Levels (ng/mL) & $3571.80)$ & $4097)$ & $0.014^{*}$ \\
Serum NSE & $386.6(45.1-$ & $397,75(99.7-$ & \\
Levels (ng/mL) & $447.6)$ & $447.2)$ & 0.446 \\
Serum GFAP & $23.9(3.9-3.0)$ & $17(2.8-31.8)$ & 0.205 \\
Levels (ng/mL) & & & \\
& & & \\
& $* \mathrm{p}<0.05$. &
\end{tabular}

the neurological clinical outcome changes, while the lower the NSE level, the better the changes according to Spearman correlation diagram (data is not shown).

To determine the power of association of S100B levels and the improvement of clinical outcome in ischemic stroke patients, we performed the receiver operating characteristic (ROC) curve analysis to find out cut off of serum S100B levels, then the association between S100B and clinical outcome was analyzed using Chi-square test. According to the ROC curve analysis, we used the cut off 608 $\mathrm{ng} / \mathrm{mL}$, then categorized the subjects into two groups, first group is subjects with serum S100B level $608 \mathrm{ng} / \mathrm{mL}$ or less and the second group is subjects with S100B level more than or $608 \mathrm{ng} / \mathrm{mL}$. The results of this analysis are depicted in table 3 . Table 3 showed that there is a significant association between serum S100B levels and the improvement of clinical outcome. The patients who have serum S100B level $608 \mathrm{ng} / \mathrm{mL}$ or less have a chance to get improved clinical outcome are six times compared to the patients who have serum S100B level more than $608 \mathrm{ng} / \mathrm{mL}$. 
Table 3: The comparison of serum S100B levels and the improvement of clinical outcome.

\begin{tabular}{|c|c|c|c|c|}
\hline \multirow{3}{*}{ Variable } & \multicolumn{2}{|c|}{ Clinical Outcome } & \multirow{3}{*}{$\mathbf{p}$} & \multirow{3}{*}{$\begin{array}{c}\text { Hazard Ratio } \\
\text { (95\% } \\
\text { Confidence } \\
\text { Interval) }\end{array}$} \\
\hline & Improved & Not improved & & \\
\hline & $(n=18)$ & $(n=24)$ & & \\
\hline \multicolumn{3}{|c|}{ Serum S100B Level } & \multirow{3}{*}{$0,007^{*}$} & \\
\hline $\begin{array}{l}\leq 608 \mathrm{ng} / \\
\mathrm{mL}\end{array}$ & $12(66.7)$ & $6(25.0)$ & & (1.560- \\
\hline $\begin{array}{l}>608 \mathrm{ng} / \\
\mathrm{mL}\end{array}$ & $6(33.3)$ & $18(75.0)$ & & 23.072) \\
\hline
\end{tabular}

\section{Conclusion}

Finally, we found that there is a significant association between the third-day onset serum S100B level and the improvement of clinical outcome, but no for NSE, GFAP levels among acute ischemic stroke patients.

\section{Funding Sources}

Nil.

\section{Acknowledgments}

We thank Dr. Putri Rachmawati Dewi from the Department of Pulmonology and Cardiovascular, Wongsonegoro Hospital Semarang Indonesia for editing a draft of this manuscript.

\section{Author Contribution}

All authors have a similar contribution to this work.

\section{Conflict of Interest}

There is no conflict of interest.

\section{Bibliography}

1. Guo Y., et al. "Pathophysiology and Biomarkers in Acute Ischemic Stroke - A Review". Tropical Journal of Pharmaceutical Research 12.6 (2013): 1097-1105.

2. Ramchandra P., et al. "Study of Neuron-Specific Enolase as Potential Biomarker for Assessing the Severity and Outcome in Patients with Cerebrovascular Accidents". Global Journal of Medical Research: A Neurology and Nervous System 14.3 (2014): 1-7.
3. Stanca DM., et al. "Plasmatic markers for early diagnostic and treatment decisions in ischemic stroke". Journal of Medicine and Life 8 (2015): 21-25.

4. Glushakova OY., et al. "Biomarkers for acute diagnosis and management of stroke in neurointensive care units". Brain Circulation 2.1 (2016): 28-47.

5. Saenger AK and Christenson RH. "Stroke biomarkers: progress and challenges for diagnosis, prognosis, differentiation, and treatment". Clinical Chemistry 56.1 (2020): 21-33.

6. Dassan P., et al. "Criteria for a clinically informative serum biomarker in acute ischaemic stroke: a review of S100B". Cerebrovascular Disease 27.3 (2009): 295-302.

7. Kulbe JR and Geddes JW. "Current status of fluid biomarkers in mild traumatic brain injury". Experimental Neurology 275 (2016): 334-352.

8. Iłżecki M., et al. "Serum Neuron-Specific Enolase as a Marker of Brain Ischemia-Reperfusion Injury in Patients Undergoing Carotid Endarterectomy". Acta clinica Croatica 55.4 (2016): 579-584.

9. Mayer CA., et al. "Blood levels of glial fibrillary acidic protein (GFAP) in patients with neurological diseases". PLoS One 8.4 (2013): e62101.

10. Luger S., et al. "Glial Fibrillary Acidic Protein Serum Levels Distinguish between Intracerebral Hemorrhage and Cerebral Ischemia in the Early Phase of Stroke'". Clinical Chemistry 63.1 (2017): 377-385.

11. Vizin T and Kos J. "Gamma-enolase: a well-known tumour marker, with a less-known role in cancer". Radiology Oncology 49.3 (2015): 217-226.

12. Hajduková L., et al. "Biomarkers of Brain Damage: S100B and NSE Concentrations in Cerebrospinal Fluid--A Normative Study". Biomed Research International 2015 (2015): 379071.

13. Cheng F., et al. "The prognostic value of serum neuron-specific enolase in traumatic brain injury: systematic review and meta-analysis". PLoS One 9.9 (2014): e106680. 
14. Hrytsiuk TD. "Serum Level of Neuron-Specific Enolase in Patients with Past Ischemic Stroke". Archives of Clinical and Medical 22.1 (2016): 1-7.

15. Anand N and Stead LG. "Neuron-specific enolase as a marker for acute ischemic stroke: a systematic review". Cerebrovascular Disease 20.4 (2005): 213-219.

16. Grube MM., et al. "Influence of acute complications on outcome 3 months after ischemic stroke". PLoS One 8.9 (2013): e75719.

17. Kwakkel G., et al. "Predictive value of the NIHSS for ADL outcome after ischemic hemispheric stroke: does timing of early assessment matter?" Journal of the Neurological Sciences 294.1-2 (2010): 57-61.

Volume 4 Issue 7 July 2021

(C) All rights are reserved by Retnaningsih., et al. 